\title{
Prologused To Represent And Reason QuAlitATIVElyOVER A SPACE DOMAIN
}

\author{
Eduardo Eloy Loza-Pacheco ${ }^{1}$, Miguel Jesús Torrez-Ruiz ${ }^{2}$ and Giovanni Guzmán- \\ Lugo $^{2}$, Dulce Loza-Pacheco ${ }^{3}$, Rodrigo Cadena-Martínez ${ }^{4}$ \\ ${ }^{1}$ Department of Applied Mathematics and Computer Science, National \\ Autonomous University of México, Mexico \\ ${ }^{2}$ Centre for Research of Computer, National Polytechnic Institute, Mexico City, \\ Mexico \\ ${ }^{3}$ Centre for Research and Advance Studies of the National Polytechnic Institute \\ ${ }^{4}$ Universidad Tecnológica de México, Mexico City, Mexico
}

\begin{abstract}
Spatial reasoning is a relevant topic in artificial intelligence with applications in geographical Information System, robotics, content-based image retrieval, traffic engineering. Additionally formal representation of knowledge allows the processing in a computer. Prolog is a programming language used in artificial intelligence that is useful to represent knowledge and perform a search, by asking questions in the knowledge base. Prolog can be used to develop a variety of applications like check the consistency or to perform any kind of reasoning. This article proposes the use of Prolog as a representation model and a reasoning engine to describe the topological relations between several objects in a geographic space, using the RCC model. The application of this simplifies the constructionprogram, allows us to focus on the spatial problem.
\end{abstract}

\section{KEYWORDS}

Knowledge based systems, Prolog, RCC model, topology, AI languages, Programming languages, spatial reasoning, spatial representation.

\section{INTRODUCTION}

Prolog is an Artificial Intelligence (AI) language that can be used for applications of symbolic computation like relational databases, mathematic logic [26], abstract problem solving, understanding natural language, design automation, symbolic equation solving, biochemical structure analysis and other areas of artificial intelligence [1,23]. Today it is difficult to find applications for Prolog. The language is often used just for teaching AI topics, Discrete Mathematics or related subjects. Today there are efforts to develop platforms to execute Prolog in a multithreaded environment, in order to enhance its performance [25].

In [2] we can find that the elements of a Knowledge Based System. We can find an Inference engine, a user interface and a knowledge base as we can see in. Additionally, in $[3,4]$. We can see that the difference between an expert system and a Shell is only the knowledge base, where the shell has no knowledge. Prolog has some characteristics that can be used to build acknowledge based system and serve as an interface between the search engine and the user interface. 


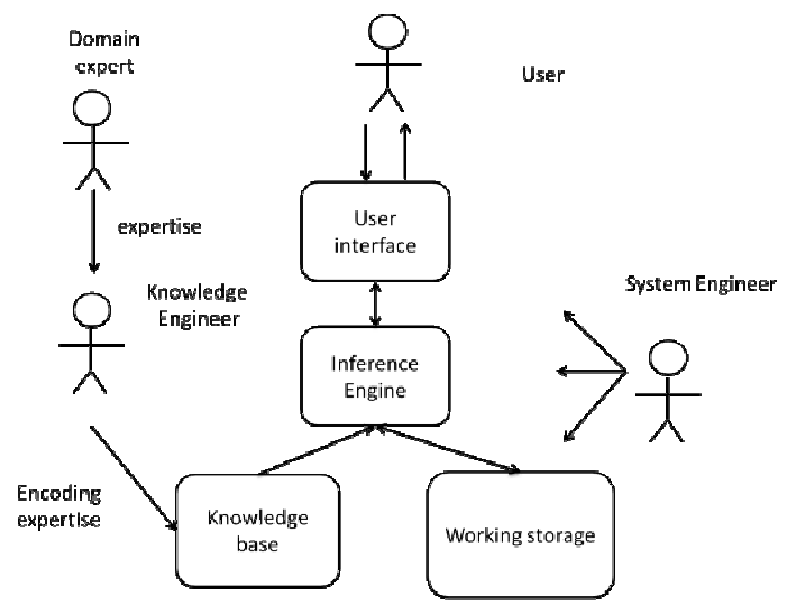

Fig. 1 Component of a Knowledge base system [3].

Prolog have a wide application as an Automated Theorem-proving [20]. For example as we can see in [21], it can be used to perform a Case Based Reasoning to construct a representation to define the properties of a waste treatment in order to know the similarities between the cases. In [24] it is used to solve a traditional AI problem that is the SAT solvers [20,24] and in [27] shows an application performing queries on ontologies with the OWL ${ }^{1}$ standard. Finally we can see the importance of Prologwether we saw the different variantsof this language like Golog [20], the extension Bousi-Prolog [28] and some implementations in Java and frameworks [29,30].

In this article, we show how Prolog can be used to define rules over a space and determine the consistency in a knowledge base. Section 2, contains some theorical considerations about topologic spatial relations and information about the RCC model. Section 3 Describes a spatial domain, where element in spaces can be categorized and recognized. Section 4, shows how Prolog and the RCC model are related. Finally Section 4 contains a conclusion about this work.

\section{TheORICAL CONSIDERATIONS}

\subsection{QUALITATIVE REASONING AND REPRESENTATION}

Qualitative spatial reasoning and representation, are widely used in several areas of artificial intelligence, for example in Geographic Information Systems, planning, robotic navigation, natural language process, visual languages, content image retrieval, traffic engineering, computer networks, 3d modelling, virtual reality [6-10].In Artificial Intelligence, we can see that one of the task of an intelligent agent is to perform a spatial reasoning with the intention of solve a problem [11]. One of the main goals of the spatial reasoning is: provide a symbolic language closer to the common sense of human beings [12] and reasoning with incomplete information, we can see and example of fuzzy logic in the spatial domainin [22]. On the other hand knowledge representation allows to have mechanisms to identify important elements of the space such as topology, orientation, shape, size and distance [13], which are the essential properties to describe the relations between objects in the space [14].

We can identify different formalisms to relate geographic objects, one of these are the topologic relations, like the 9-intersection model of Egenhofer [15], the RCC model of Cohn [16], and their

\footnotetext{
${ }^{1}$ Ontology Web Language. https://www.w3.org/OWL/
} 
variations such as RCC5, RCC23 [17]. RCC model and 9-intersection, has become one of the most popular models to describe binary topological qualitative binary relations [18].

\subsection{THE RCC8 MODEL}

The RCC model describe Euclidian regions or topological between two objects. In the next figure we can see the relations of the model [19]

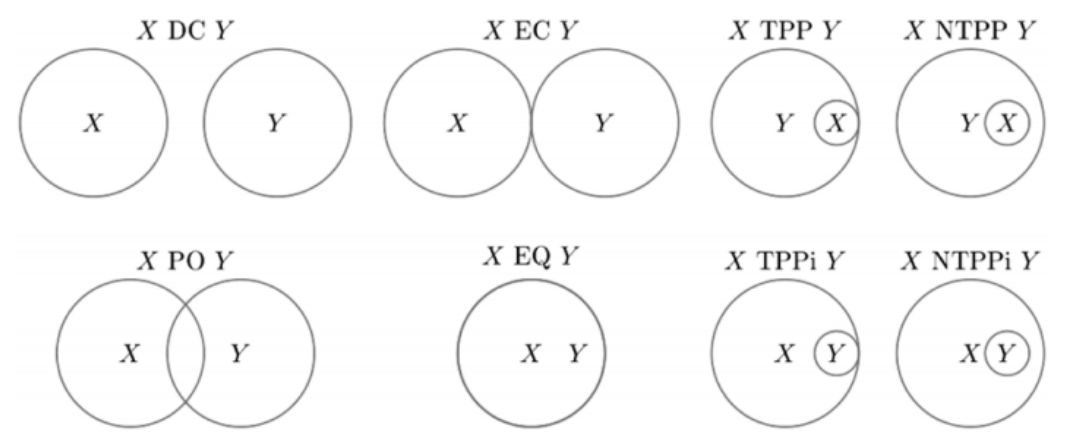

Figure2. RCC8 model

The relations of the model are.

- Disconnected (DC)

- Externally Connected (EC)

- Tangential Proper Part (TPP)

- Non-Tangential Proper Part (NTPP)

- Partially Overlapping (PO)

- Equally Connected (EC)

- Tangential Proper Part Inverse (TPPi)

- Non-Tangential Proper Part Inverse (NTPPi)

\section{DESCRIBING A DOMAIN}

I order to use Prologto reason over a space domain, closely as a way the human thinks.In the space domain, we need to define some spatial relations like directional relations and topological relations, for example as we can see in the following image.

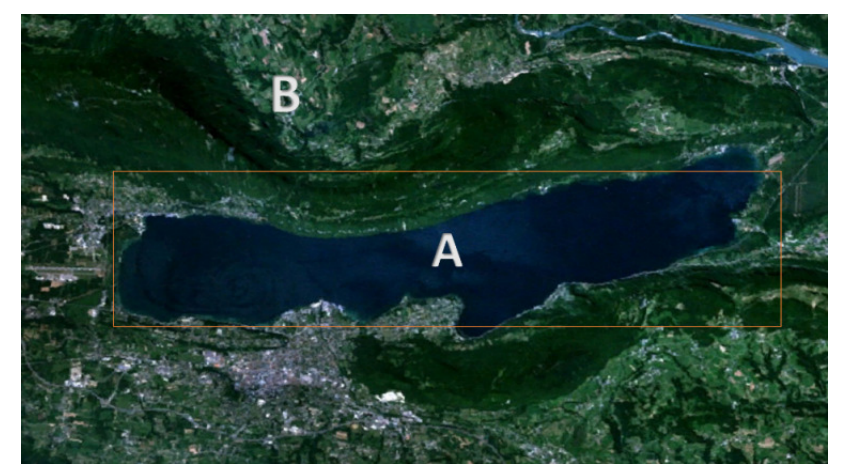

Fig. 3 Lake of Bourget 
If we can detect the objects A and B,by using any kind of segmentation image. For these purposes we will identify A as a body of water and B as a green area. Having defined the two objects we need to define the spatial relation between them and the spatial relation in this case is a topological one. In the next propositions we can express this knowledge.

We can have the following sets of facts.

A is blue

$B$ is green

So we can have three rules

If $A$ is blue, then is a body of water.

If $B$ is green, then is forest.

If $B$ surrounds $A$, then $A$ is a lake

We can see the previous facts and rules in Prologin the following in figure

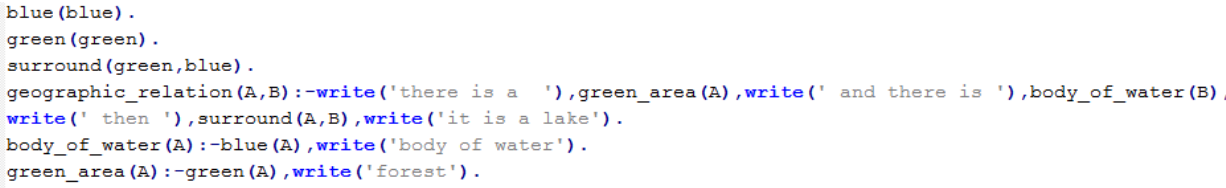

Figure 4. Identified elements.

If we ask for the relation of them

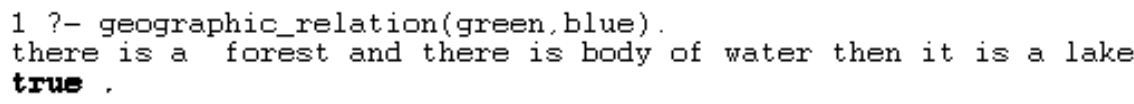

Also we may have another implementation in the space like the image below.

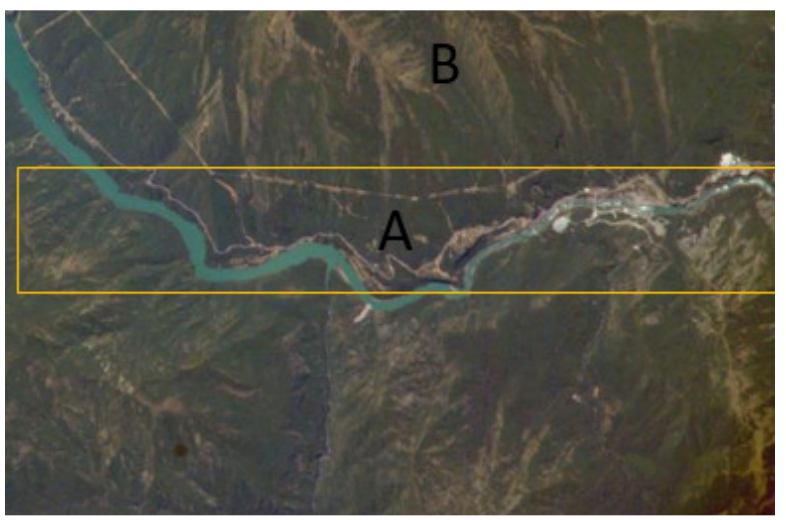

Figure5. Identified elements.

We just need to extend our Knowledge base with the following rules and change the facts. Of course, the granularity we apply to our domain, the more punctual answers we obtain.

Blue is a body of water

Green is a forrest

$B$ is external/surronds $A$ then $A$ is a lake and $B$ and $A$ conforms a forest

$B$ cross $A$ then $A$ is a river.

River is a body of water. 
In Prolog can be seen like this (Figure 6).

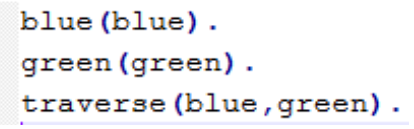

Fig 6. Facts of the elements.

And we can construct the rules of inference, with the intention to define, the spatial relation of A and $\mathrm{B}$.

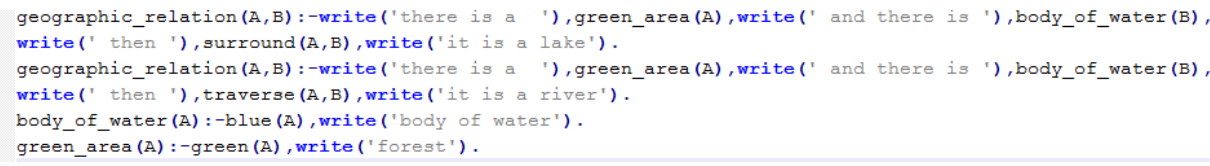

Fig. 7 Rules of Inference.

\section{Applying PROLOG To The Space Domain Using The RCC Model 4.1IDENTIFYING The RELATION OBJECTS USING THE RCC MODEL}

Suppose we have the following set of objects from A to F, as described in the figure 1 . We want to obtain the relations between them.

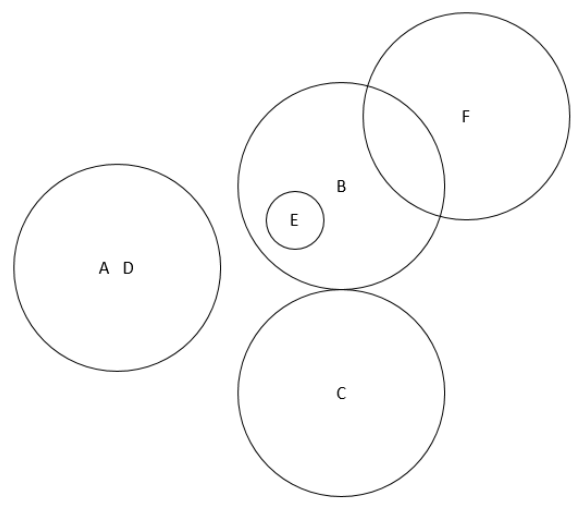

Fig 8. A set of five objects

We need to have an appropriate representation of the objects. For example we can represent them in Prolog as predicates (As show in figure 2).

$$
\begin{aligned}
& d c(a, b) . \\
& \text { ec }(b, c) . \\
& \text { eqq }(a, d) . \\
& \text { po }(f, b) . \\
& p p(b, e) .
\end{aligned}
$$

Fig 9. Set of five facts that represent a space domain.

Where the description is the following: dc denotes a is disconnected from $b$, ec denotes $b$ is externally connected with c, eqq denotes a is equally connected with d, po f partially overlaps $b$ 
and $\mathrm{pp} \quad \mathrm{b}$ tangential proper part e.Then, if we can obtain the relations between several objectsProlog can be used to describe the relation, we can just search the relations between all the elements in our knowledge base. Then to generalize, for every object that we can read, we can define the rules we see in figure 10.

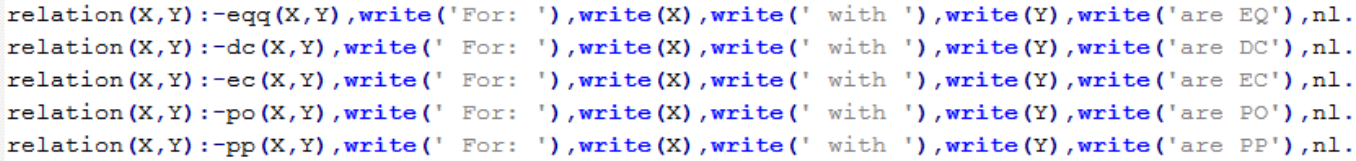

Figure 10. Definition of the relations.

Having load the knowledge base, and the rules. We can ask Prolog about the relations in the knowledge base by writing the following questions.

?-relation(X,Y).

The results of the search we obtain is the relations between all the elements in the knowledge base. As we can see in the in the figure 11 .

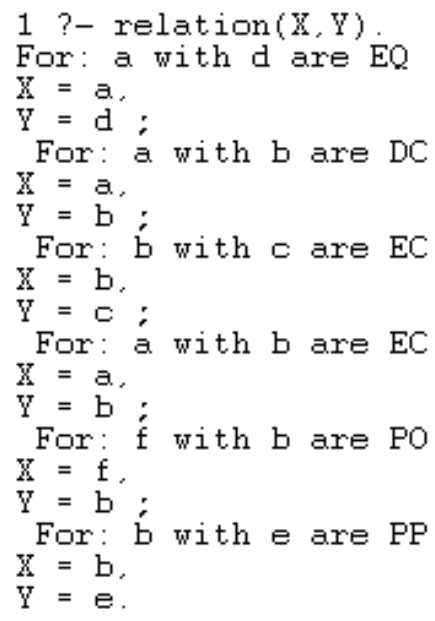

Figure 11. Results of the question.

\subsection{Checking The Consistency}

To verify the consistency between all elements in the knowledge base we add a predicate we can see these changes in line two and three. As we see it is not possible for the objects $a$ and $b$ to be disconnected and externally connected. The conclusion is that it must be an error in the knowledge base.

$$
\begin{aligned}
& \text { dc }(a, b) . \\
& \text { ec }(a, b) . \\
& \text { ec }(b, c) . \\
& \text { eqq }(a, d) \text {. } \\
& \text { po }(f, b) . \\
& \text { pp }(b, e) .
\end{aligned}
$$

Figure 12. Knowledge base with inconsistency errors. 
To check the consistency we can use a conjunction to find if there is an error in the knowledge base.

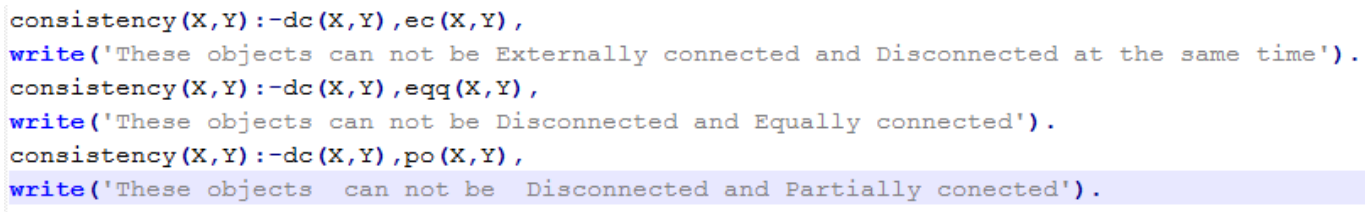

Figure 13. Rules for consistency.

For example, we can define that a relations between two objects like A and A since are the same, we can omitted. As we can see in the code, no other object in the relations have the same consistency error as we define so we obtain false.

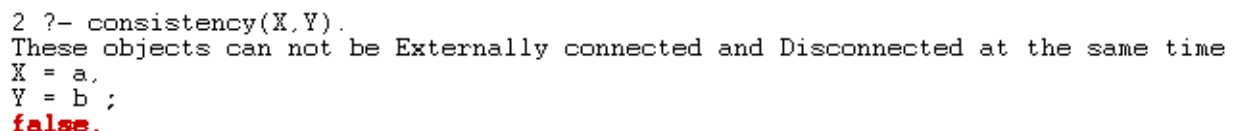

\section{Conclusions}

In Prolog define relations between a pair of objects does not requires much complexity, it is just requires the facts and the rules of the space domain. Also the representation of knowledge it is closer as the human common sense. To perform reasoning we simply need to ask about the objects related in the knowledge base and Prolog performs the reasoning.Additionally Prolog allows to check the consistency so we can evaluate if there is an error identifying objects. The knowledge base accepts add more knowledge and can be processed all the information again.So in sum Prolog can describe all the objects in the space.Prolog can be integrated to a variety of applications as a shell of and knowledge base, whether there are defined the objects and their relations.

\section{ACKNOWLEDGEMENTS}

The authors would like to thank to the National Autonomous University of México and the Department of Applied Mathematics and Computer Science,The"Consejo Nacional de Ciencia y Tecnologia" of Mexico.

\section{REFERENCES}

[1] Clocksin, W., and Mellish C. S., "Programming in PROLOG with the ISO standard", Springer, 2003.

[2] Krishnamoorthy, C. S., and Rajeev, S., "Artificial intelligence and expert systems for engineers", CRC Press, Vol. 11, 1996.

[3] Merritt, D., "Building expert systems in Prolog", Springer, 1989.

[4] Coppin, B., "Artificial intelligence illuminated", Jones \& Bartlett Learning, 2004.

[5] Schlenoff, C., Pietromartire, A., Kootbally, Z., Balakirsky, S., \&Foufou, S., Ontology-based state representations for intention recognition in human-robot collaborative environments, Robotics and Autonomous Systems, 61(11), 1224-1234. 2013.

[6] W. Shengsheng, L. Yiting, L. Dayou, B. Dickson, X. Wang, "Multigranularity and metric spatial reasoning”, Expert System with Applications, Vol. 41, Elsevier, pp. 3116-3133.Robotics and Autonomous Systems, Vol. 61, p.1124-1234, 2014

[7] K. William, et. al., "Spatial representation and reasoning for human-robot collaboration", AAAI, Vol. 7, pp. 1554-1559, 2007.

[8] A. Cohn, J. Renz, "Qualitative spatial representation and resoning", Foundations of Artificial Intelligence, Elsevier, Vol. 3, pp. 551-596, 2008 
[9] W. Lui, X. Zhang, S. Li, M. Ying, "Reasoning about cardinal directions between extended objects", Artificial Intelligence, Elsevier, Vol. 174, No. 12, pp.951-983, 2010

[10] J. Leopold, C. Sabharwal, "Spatial relations between 3D objects: The association between natural language, topology and metrics", Journal of Visual Languages, Elsevier, 2015,

[11] U. Kurup, N. Cassimatis, "Quantitative spatial reasoning for general intelligence", Proceedings of the third Conference on Artificial General Intelligence Conference, pp. 1-6, 2010.

[12] R. Moratz, D. Lücke, T. Mossaowski, “A condensed semantics for qualitative spatial reasoning about oriented straight line segments", Artificial Intelligence, Elsevier, Vol. 175, No. 16, pp. 2099-2127, 2011.

[13] A. Cohn, J. Renz, "Qualitative spatial representation and resoning", Foundations of Artificial Intelligence, Elsevier, Vol. 3, pp. 551-596, 2008

[14] D. Wolter, J. Lee, "Qualitative reasoning with directional relations", Artificial Intelligence, Elsevier, Vol. 174. No. 174, pp.1498-1507, 2010

[15] M. Egenhofer, and R. Franzosa, "Point-set topological spatial relations", International Journal of Geographical Information Science, vol. 5, no. 2, Taylor \& Francis, pp. 161-174, 1991

[16] [28] D. A. Randell, Z. Cui, and A. G. Cohn, "A spatial logic based on regions and connection”, Third International Conference on Knowledge Representation and Reasoning, vol. 92, Morgan Kaufmann, pp. 165-176, 1992.

[17] D. Papadias, and M. J. Egenhofer, "Algorithms for hierarchical spatial reasoning”, GeoInformatica, vol. 1, no. 3, Springer, pp. 1-2, 1997.

[18] M. Egenhofer, M. Dube, "Topological relations from metric refinements", Proceeding of the 17th ACM SIGSPATIAL International Conference on Advances in Geographic Information Systems, ACM, pp. 158-167, 2009.

[19] C. Schenoff, A. Pietromartire, K. Zeid, S. Balakirsky, S. Foufou, "Ontology-based state representations for intention recognition”, Robotic and Autnomous Systems, Elsevier, Vol. 61, N. 11, pp. 1224-1234, 2013

[20] R. Brachman \& H. Levesque, Knowledge Representation and Reasoning, The Morgan Kaufmann Series in Artificial Intelligence, Cap. 5, 2004.

[21] P. Chazara, S. Negny, L. Montastruc, Flexible knowledge representation and new similarity measure: Application on case based reasoning for waste treatment, Expert Systems with Applications, 58, 143154, 2016.

[22] L. Hu, N. Xiong, F. deng, K. Yan \& L. Lui, A Fuzzy Description Logic Based Spatial Direction Reasoning Approach. Journal of Residuals Science \& Technology, 13(8), 2016.

[23] G. Li, Y. Shao, S. Hong \& B. Liu, An IPC-based Prolog design pattern for integrating backward chaining inference into applications or embedded systems. Chinese Journal of Aeronautics, 27(6), 1571-1577, 2014.

[24] J. Howe \& A. King, A pearl on SAT and SMT solving in Prolog. Theoretical Computer Science, 435, 43-55, 2012

[25] M. Areias\& R. Rocha, on scaling dynamic programming problems with a multithreaded tabling Prolog system, Journal of Systems and Software, 2016.

[26] A. Csenki, Enigma 1225: Prolog-assisted solution of a puzzle using discrete mathematics. Computers \& Mathematics with Applications, 52(3-4), 383-400, 2006

[27] J. Almendros-Jimenez, A prolog-based query language for OWL, Electronic Notes in Theoretical Computer Science, 271, 3-22, 2011

[28] P. Julián-Iranzo\& C. Rubio-Manzano, A sound and complete semantics for a similarity-based logic programming language, Fuzzy Sets and Systems, 317, 1-26. 2017.

[29] A. Amandi, M. Campo \& A. Zunino, JavaLog: a framework-based integration of Java and Prolog for agent-oriented programming. Computer languages, systems \& structures, 31(1), 17-33, 2005.

[30] E. Denti, A. Omicini\& A. Ricci, Multi-paradigm Java-Prolog integration in tuProlog. Science of Computer Programming, 57(2), 217-250, 2005. 


\section{AUTHORS}

Eduardo E. Loza-Pacheco $\mathrm{He}$ is a Professor of the National Autonomous University of México at the Department of Applied Mathematics and Computer Science. He is interested in artificial intelligence, specifically Knowledge based system, parallel programming and information se curity.

Giovanni Guzman-Lugo He is a researcher at the Centre for Research of Computer, National Polytechnic Institute. He is interested in artificial intelligence, pattern recognition.

Miguel Torres-Ruiz He is a Researcher at The Centre for Research of Computer, National Polytechnic Institute. He is interested in semantic web, spatial reasoning, computational geometry and geoprocessing.

Dulce Loza-Pacheco She is a researcher at the Centre for Research and Advance Studies of the National Polytechnic Institute. He is interested in topics semantic web, artificial intelligence, software engineering.

Rodrigo Cadena-Martinez $\mathrm{He}$ is a professor at Technologic University of Mexico. He is interested in topics semantic similarity and ontologies. 